\title{
Uniform Lower Bound for Intersection Numbers of $\psi$-Classes
}

\author{
Vincent DELECROIX ${\dagger^{\dagger}}^{1}$, Élise GOUJARD ${ }^{\dagger^{2}}$, Peter ZOGRAF ${\dagger^{3} \dagger^{4}}^{2}$ and Anton ZORICH ${\dagger^{5} \dagger^{6}}^{6}$ \\ $\dagger^{1}$ LaBRI, Domaine universitaire, 351 cours de la Libération, 33405 Talence, France \\ E-mail: 20100.delecroix@gmail.com \\ $\dagger^{2}$ Institut de Mathématiques de Bordeaux, Université de Bordeaux, \\ 351 cours de la Libération, 33405 Talence, France \\ E-mail: elise.goujard@gmail.com \\ $\dagger^{3}$ Steklov Mathematical Institute, Fontanka 27, St. Petersburg 191023, Russia \\ E-mail: zograf@pdmi.ras.ru \\ $\dagger^{4}$ Chebyshev Laboratory, St. Petersburg State University, \\ 14th Line V.O. 29B, St. Petersburg, 199178, Russia \\ $\dagger^{5}$ Center for Advanced Studies, Skoltech, Russia \\ E-mail: anton.zorich@gmail.com \\ $\dagger^{6}$ Institut de Mathématiques de Jussieu - Paris Rive Gauche, Bâtiment Sophie Germain, \\ Case 7012, 8 Place Aurélie Nemours, 75205 PARIS Cedex 13, France
}

Received April 09, 2020, in final form August 21, 2020; Published online August 26, 2020 https://doi.org/10.3842/SIGMA.2020.086

\begin{abstract}
We approximate intersection numbers $\left\langle\psi_{1}^{d_{1}} \cdots \psi_{n}^{d_{n}}\right\rangle_{g, n}$ on Deligne-Mumford's moduli space $\overline{\mathcal{M}}_{g, n}$ of genus $g$ stable complex curves with $n$ marked points by certain closedform expressions in $d_{1}, \ldots, d_{n}$. Conjecturally, these approximations become asymptotically exact uniformly in $d_{i}$ when $g \rightarrow \infty$ and $n$ remains bounded or grows slowly. In this note we prove a lower bound for the intersection numbers in terms of the above-mentioned approximating expressions multiplied by an explicit factor $\lambda(g, n)$, which tends to 1 when $g \rightarrow \infty$ and $d_{1}+\cdots+d_{n-2}=o(g)$.
\end{abstract}

Key words: intersection numbers; $\psi$-classes; Witten-Kontsevich correlators; moduli space of curves; large genus asymptotics

2020 Mathematics Subject Classification: 14C17; 14H70

Dedicated to D.B. Fuchs

on the occasion of his 80th birthday

\section{Introduction}

Let $\overline{\mathcal{M}}_{g, n}$ be the Deligne-Mumford moduli space of genus $g$ complex stable algebraic curves (possibly with nodes), with $n>0$ distinct labeled marked points. Consider the tautological line bundles $\mathcal{L}_{i} \rightarrow \overline{\mathcal{M}}_{g, n}, i=1, \ldots, n$, defined fiberwise by $\left.\mathcal{L}_{i}\right|_{C, x_{1}, \ldots, x_{n}} \cong T_{x_{i}}^{*} C$, where $C$ is a genus $g$ curve with marked points $x_{1}, \ldots, x_{n}$ (the definition makes sense since the marked points are not allowed to coincide with the nodes).

In the late 1980-ies E. Witten [21] introduced a theory of two-dimensional topological gravity, where the classes $\psi_{i}=c_{1}\left(\mathcal{L}_{i}\right), i=1, \ldots, n$, played the role of observables, and the intersection

This paper is a contribution to the Special Issue on Algebra, Topology, and Dynamics in Interaction in honor of Dmitry Fuchs. The full collection is available at https://www.emis.de/journals/SIGMA/Fuchs.html 
numbers

$$
\left\langle\tau_{d_{1}} \cdots \tau_{d_{n}}\right\rangle_{g, n}=\left\langle\psi_{1}^{d_{1}} \cdots \psi_{n}^{d_{n}}\right\rangle_{g, n}=\int_{\overline{\mathcal{M}}_{g, n}} \psi_{1}^{d_{1}} \cdots \psi_{n}^{d_{n}},
$$

where $d_{1}+\cdots+d_{n}=3 g-3+n$, represented correlators of the theory. Following a common convention, we will omit $g$ and $n$, or just $n$ when they are clear from the context. On the basis of a low genus evidence and considerations from physics, E. Witten conjectured that the generating function

$$
F\left(t_{0}, t_{1}, \ldots\right)=\sum_{\substack{g \geq 0 \\ n \geq 1}} \frac{1}{n !} \sum_{\substack{d_{1}+\cdots+d_{n} \\=3 g-3+n}}\left\langle\tau_{d_{1}} \cdots \tau_{d_{n}}\right\rangle t_{d_{1}} \cdots t_{d_{n}}
$$

(total free energy of Witten's two-dimensional topological gravity) satisfies the KdV (Kortevegde Vries) hierarchy. An equivalent formulation of Witten's conjecture is that the partition function $e^{F}$ is a highest weight vector of a Virasoro algebra representation, see papers [9, 10] of R. Dijkgraaf and of R. Dijkgraaf, H. Verlinde, and E. Verlinde. Witten's conjecture was first proven by M. Kontsevich [16], and then several other proofs appeared, including the proofs due to A. Okounkov and R. Pandharipande [20], to M. Kazarian and S. Lando [15], to M. Mirzakhani $[18,19]$. Note that the two-dimensional topological gravity may be interpreted as the simplest instance of the Gromov-Witten theory, where the tagret space is a point.

Computability of the intersection numbers $\left\langle\tau_{d_{1}} \cdots \tau_{d_{n}}\right\rangle$ is an important open problem. Besides the cases of small $g$ or $n$ no general explicit formulas for the numbers $\left\langle\tau_{d_{1}} \cdots \tau_{d_{n}}\right\rangle_{g, n}$ are known. In applications it is often sufficient to know large genus behavior of these intersection numbers, and here either not much was known in general before the current paper and before even more recent beautiful work by A. Aggarwal [3].

In this note we prove a simple lower bound for the numbers that appears to be asymptotically exact as $g \rightarrow \infty$. To begin with, we recall some basic facts about the numbers $\left\langle\tau_{d_{1}} \cdots \tau_{d_{n}}\right\rangle$ (Witten's correlators). They are uniquely defined by the initial data

$$
\left\langle\tau_{0}^{3}\right\rangle=1, \quad\left\langle\tau_{1}\right\rangle=\frac{1}{24}
$$

via the recursive relations known as Virasoro constraints that we present below.

Virasoro constraints (in Dijkgraaf-Verlinde-Verlinde form $[9,10]$ ):

$$
\begin{aligned}
\left\langle\tau_{k+1} \tau_{d_{1}} \cdots \tau_{d_{n}}\right\rangle_{g}= & \frac{1}{(2 k+3) ! !}\left[\sum_{j=1}^{n} \frac{\left(2 k+2 d_{j}+1\right) ! !}{\left(2 d_{j}-1\right) ! !}\left\langle\tau_{d_{1}} \cdots \tau_{d_{j}+k} \cdots \tau_{d_{n}}\right\rangle_{g}\right. \\
& +\frac{1}{2} \sum_{\substack{r+s=k-1 \\
r, s \geq 0}}(2 r+1) ! !(2 s+1) ! !\left\langle\tau_{r} \tau_{s} \tau_{d_{1}} \cdots \tau_{d_{n}}\right\rangle_{g-1} \\
& +\frac{1}{2} \sum_{\substack{r+s=k-1 \\
r, s \geq 0}}(2 r+1) ! !(2 s+1) ! ! \\
& \left.\times \sum_{\{1, \ldots, n\}=I \amalg J}\left\langle\tau_{r} \prod_{i \in I} \tau_{d_{i}}\right\rangle_{g^{\prime}}\left\langle\tau_{s} \prod_{i \in J} \tau_{d_{i}}\right\rangle_{g-g^{\prime}}\right] .
\end{aligned}
$$

For $k=-1$ and $k=0$ the above relations have particularly simple form.

String equation $(k=-1)$ :

$$
\left\langle\tau_{0} \tau_{d_{1}} \cdots \tau_{d_{n}}\right\rangle_{g, n+1}=\left\langle\tau_{d_{1}-1} \cdots \tau_{d_{n}}\right\rangle_{g, n}+\cdots+\left\langle\tau_{d_{1}} \cdots \tau_{d_{n}-1}\right\rangle_{g, n} .
$$


Dilaton equation $(k=0)$ :

$$
\left\langle\tau_{1} \tau_{d_{1}} \cdots \tau_{d_{n}}\right\rangle_{g, n+1}=(2 g-2+n)\left\langle\tau_{d_{1}} \cdots \tau_{d_{n}}\right\rangle_{g, n} .
$$

For any partition $\boldsymbol{d}$ of $3 g-3+n$ into a sum of $n$ nonnegative integers define $\varepsilon(\boldsymbol{d})$ through the following equation:

$$
\left\langle\psi_{1}^{d_{1}} \cdots \psi_{n}^{d_{n}}\right\rangle_{g, n}=\frac{(6 g-5+2 n) ! !}{\left(2 d_{1}+1\right) ! ! \cdots\left(2 d_{n}+1\right) ! !} \cdot \frac{1}{g ! \cdot 24^{g}} \cdot(1+\varepsilon(\boldsymbol{d})) .
$$

We denote by $\Pi(m, n)$ the set of ordered partitions of an integer $m$ into a sum of $n$ nonnegative integers.

Main Conjecture ([7]). For any strictly positive constant $C$

$$
\lim _{g \rightarrow+\infty} \max _{1 \leq n \leq C \log (g)} \max _{\boldsymbol{d} \in \Pi(3 g-3+n, n)}|\varepsilon(\boldsymbol{d})|=0 .
$$

Corollary 6 below makes the first step towards a proof of the Main Conjecture. It establishes an efficient uniform lower bound for $\varepsilon(\boldsymbol{d})$ for those partitions $\boldsymbol{d}$ for which the sum of the first $n-2$ entries is small with respect to the sum of the remaining two entries.

Remark 1. It follows from the definition of $\varepsilon(\boldsymbol{d})$ that $\varepsilon(\boldsymbol{d})$ does not change under any permutation of the entries of $\boldsymbol{d}$.

Remark 2. It is plausible, that much stronger statement might be true, where the bound $n<C \log (g)$ is replaced by the bound $n<g^{\alpha}$ with any fixed $\alpha$ satisfying $\alpha<\frac{1}{2}$. The reason why one cannot go beyond the bound $n<\sqrt{g}$ is explained at the very end of Section 1 .

Remark 3 (added in proofs). The Main Conjecture was proved in a very strong form in the recent paper [3] of A. Aggarwal.

Motivation. Certain universality phenomena in flat and hyperbolic geometry and in dynamics of surfaces manifest themselves in large genera. The large genus asymptotics of the Masur-Veech volumes of strata in moduli spaces of Abelian differentials conjectured in [12] was successfully proved by independent methods in [2] and in [6]. However, the analogous conjectures stated in [4] on the large genus asymptotics of the Masur-Veech volumes of strata in moduli spaces of quadratic differentials are open.

There are several approaches to evaluation of Masur-Veech volumes of such strata. The original approach of A. Eskin and A. Okounkov discovered in [11] uses characters of the symmetric group. Using modern computers for exact computations based on this approach, É. Goujard evaluated in [13] the volumes of all strata up to dimension 11. Currently it is not known how to obtain volume asymptotics based on this approach.

The recent paper [5] of D. Chen, M. Möller and A. Sauvaget expressed the Masur-Veech volume of the principal stratum through certain very special linear Hodge integrals. The papers [14] of M. Kazarian and [22] of D. Yang, D. Zagier and Y. Zhang provide extremely efficient recursive formulae for these Hodge integrals, which allow to compute exact values of the volumes of the principal strata up to genus 250 and more. However, currently it is not known how to prove large genus asymptotic formulae for the Masur-Veech volume of the principal stratum developing this approach.

One more approach to evaluation of Masur-Veech volumes is elaborated in our paper [7] where we express the Masur-Veech volume of the principal stratum of meromorphic quadratic differentials with at most simple poles through intersection numbers of $\psi$-classes and suggest conjectures aimed to prove large genus asymptotics of these volumes. This conjectural scheme involves the Main Conjecture stated above as one of the key ingredients. 
Remark 4 (added in proofs). The asymptotic formula for the Masur-Veech volume conjectured in [7] was recently proved in [3] by A. Aggarwal. Together with the Main Conjecture proved by A. Aggarwal in the same paper, this allowed us to provide in [8] a detailed description of the asymptotic geometry of random square-tiled surfaces and of random simple closed multicurves on surfaces of large genus.

\subsection{State of the art}

Currently we have the following evidence towards the Main Conjecture. Direct computation shows that $\varepsilon(0,0,0)=0$. It is known [21] that

$$
\left\langle\tau_{3 g-2}\right\rangle_{g, 1}=\frac{1}{24^{g} \cdot g !}
$$

so for all 1-correlators we have

$$
\varepsilon(3 g-2)=0 .
$$

Applying the string equation recursively we get

$$
\left\langle\tau_{0}^{n-1} \tau_{3 g-3+n}\right\rangle_{g, n}=\frac{1}{24^{g} \cdot g !},
$$

so for all partitions with at most one nonzero entry we have

$$
\varepsilon\left(0^{n-1}, 3 g-3+n\right)=0 .
$$

For 2-correlators the Main Conjecture is valid. Namely, by Remark 1, we have $\varepsilon\left(d_{1}, d_{2}\right)=$ $\varepsilon\left(d_{2}, d_{1}\right)$ for any $\left(d_{1}, d_{2}\right) \in \Pi(3 g-1,2)$. Thus, we may assume that $d_{1}<d_{2}$. We have already seen that $\varepsilon(0,3 g-1)=0$. For the remaining 2-partitions we have the following bounds:

Theorem ([7]). For all $g \in \mathbb{N}$ and for all integer $k$ satisfying $2 \leq k \leq \frac{3 g-1}{2}$ the following bounds are valid:

$$
-\frac{2}{6 g-1}=\varepsilon(1,3 g-2)<\varepsilon(k, 3 g-1-k)<0=\varepsilon(0,3 g-1) .
$$

We performed a detailed analysis of $\varepsilon(k, 3 g-1-k)$ in [7] based on [23]. In particular, for large $g$ the error term $\varepsilon(k, 3 g-1-k)$ rapidly tends to 0 when $k$ approaches $\frac{3 g-1}{2}$, so the statement of the above theorem can be seriously strengthened, if needed.

It is easy to compute $\varepsilon(\boldsymbol{d})$ explicitly for those partitions where all but one entries $d_{1}, \ldots, d_{n-1}$ are equal to 0 or 1 . Namely, we first apply recursively the dilaton equation eliminating all those entries of the partition, which are equal to 1, and then apply (4). In particular,

$$
\left\langle\tau_{1}^{n-1} \tau_{3 g-2}\right\rangle_{g, n}=(2 g-3+n)(2 g-4+n) \cdots(2 g-1) \cdot \frac{1}{24^{g} \cdot g !},
$$

so

$$
\begin{aligned}
1+\varepsilon((1,1, \ldots, 1,3 g-2)) & =1+\varepsilon\left(\left(1^{n-1}, 3 g-2\right)\right) \\
& =(2 g-3+n)(2 g-4+n) \cdots(2 g-1) \cdot \frac{3^{n-1} \cdot(6 g-3) ! !}{(6 g-5+2 n) ! !} \\
& =\frac{6 g-3+3(n-2)}{6 g-1+2(n-2)} \cdot \frac{6 g-3+3(n-3)}{6 g-1+2(n-3)} \cdots \frac{6 g-3}{6 g-1} .
\end{aligned}
$$


This implies that for any constant $\alpha$ satisfying $0<\alpha<\frac{1}{2}$ (respectively $\frac{1}{2}<\alpha$ ) we have

$$
\begin{aligned}
& \lim _{g \rightarrow+\infty} \max _{1 \leq n \leq g^{\alpha}}\left|\varepsilon\left(1^{n-1}, 3 g-2\right)\right|=0, \quad \text { when } 0<\alpha<\frac{1}{2}, \\
& \lim _{g \rightarrow+\infty} \inf _{n \geq g^{\alpha}} \varepsilon\left(1^{n-1}, 3 g-2\right)=+\infty, \quad \text { when } \frac{1}{2}<\alpha,
\end{aligned}
$$

which explains why the restriction $\alpha<\frac{1}{2}$ in Remark 2 cannot be loosened.

\section{Uniform lower bound}

Given a real number $L$ and integers $g \geq 1$ and $n \geq 3$, denote by $\Pi_{L}(3 g-3+n, n)$ the following subset of ordered partitions:

$$
\Pi_{L}(3 g-3+n, n)=\left\{\boldsymbol{d} \in \Pi(3 g-3+n, n) \mid d_{1}+\cdots+d_{n-2} \leq L\right\} .
$$

For any nonnegative $L$ and any integer $g \geq 1$ we define $\Pi_{L}(3 g-2,1)=\Pi(3 g-2,1)$ and $\Pi_{L}(3 g-1,2)=\Pi(3 g-1,2)$.

Define the following function of integer arguments $g, L$, satisfying $g>L \geq 0$ :

$$
\lambda(g, L)=\left(\prod_{i=0}^{L-1}\left(1-\frac{1}{6(g-i)+1}\right)\right) \cdot\left(1-\frac{2}{6(g-L)-1}\right),
$$

where, by convention,

$$
\lambda(g, 0)=\left(1-\frac{2}{6 g-1}\right) .
$$

It follows from the definition of $\lambda(g, L)$ that $0<\lambda(g, L)<1$ for any $g>L \geq 0$.

Theorem 5. Let $g, L$ be nonnegative integers such that $g>L$. For any partition $\boldsymbol{d} \in \Pi_{L}(3 g-$ $3+n, n)$ one has

$$
\varepsilon(\boldsymbol{d}) \geq \lambda(g, L)-1 \text {. }
$$

Corollary 6. Let $L(g)$, where $g=1,2, \ldots$, be any sequence of nonnegative integers such that $L(g)=o(g)$ as $g \rightarrow+\infty$. One has

$$
\lim _{g \rightarrow+\infty} \inf _{n \geq 1} \min _{\boldsymbol{d} \in \Pi_{L(g)}(3 g-3+n, n)} \varepsilon(\boldsymbol{d})=0 .
$$

Proof of Corollary 6. Definition (7) of $\lambda(g, L)$ implies that for any sequence $L(g)$ of nonnegative integers satisfying $L(g)=o(g)$ as $g \rightarrow+\infty$ one has

$$
\lim _{g \rightarrow+\infty} \lambda(g, L(g))=1 .
$$

Now (10) follows from combination of (9) and (3).

Remark 7. Proposition 3.2 in [17] claims that for any triple $(n, K, M)$ of positive integers one has

$$
\lim _{g \rightarrow+\infty} \max _{\boldsymbol{d} \in \Pi_{K}(3 g-3+n, n)}|\varepsilon(\boldsymbol{d})|=0
$$

under the additional requirement that $d_{n-1} \leq M$. 
We start by proving three Lemmas (corresponding to the string and the dilaton equations, and to Virasoro constraints). It would be useful to introduce the following notation. Given $\boldsymbol{d} \in \Pi(3 g-3+n, n)$ let

$$
\left\lfloor\tau_{d_{1}} \cdots \tau_{d_{n}}\right\rfloor_{g, n}=\frac{(6 g-5+2 n) ! !}{\left(2 d_{1}+1\right) ! ! \cdots\left(2 d_{n}+1\right) ! !} \cdot \frac{1}{g ! \cdot 24^{g}} .
$$

By definition of $\varepsilon(\boldsymbol{d})$ we have

$$
\left\langle\tau_{d_{1}} \cdots \tau_{d_{n}}\right\rangle_{g, n}=\left\lfloor\tau_{d_{1}} \cdots \tau_{d_{n}}\right\rfloor_{g, n} \cdot(1+\varepsilon(\boldsymbol{d})) .
$$

From now on we suppose that $g \geq 1$.

Lemma 8. Let $\boldsymbol{d} \in \Pi(3 g-2+n, n-k)$ such that $d_{j}>0$ for $j=1, \ldots, n-k$. We assume that $k \geq 0$ and $n-k>0$. Define $\delta_{\text {string }}\left(0^{k+1}, \boldsymbol{d}\right)$ by equation

$$
\begin{aligned}
& \left\lfloor\tau_{0}^{k+1} \tau_{d_{1}} \cdots \tau_{d_{n-k}}\right\rfloor_{g, n+1} \cdot\left(1+\delta_{\text {string }}\left(0^{k+1}, \boldsymbol{d}\right)\right) \\
& \quad=\left\lfloor\tau_{0}^{k} \tau_{d_{1}-1} \cdots \tau_{d_{n-k}}\right\rfloor_{g, n}+\cdots+\left\lfloor\tau_{0}^{k} \tau_{d_{1}} \cdots \tau_{d_{n-k}-1}\right\rfloor_{g, n} .
\end{aligned}
$$

Then

$$
\delta_{\text {string }}\left(0^{k+1}, \boldsymbol{d}\right)=\frac{n-k-1}{6 g-3+2 n} .
$$

In particular, for any $\boldsymbol{d}$ as above and for any $k \geq 0$ we have

$$
\delta_{\text {string }}\left(0^{k+1}, \boldsymbol{d}\right) \geq 0 \text {. }
$$

Proof. Dividing both sides of equation (13) by $\left\lfloor\tau_{0}^{k+1} \tau_{d_{1}} \ldots \tau_{d_{n-k}}\right\rfloor_{g, n+1}$ and applying definition (11) to all terms involved in the right-hand side of the resulting equation we get

$$
\begin{aligned}
1+\delta_{\text {string }}\left(0^{k+1}, \boldsymbol{d}\right) & =\frac{\left(2 d_{1}+1\right)+\cdots+\left(2 d_{n-k}+1\right)}{6 g-3+2 n} \\
& =\frac{2(3 g-2+n)+(n-k)}{6 g-3+2 n}=\frac{6 g-4+3 n-k}{6 g-3+2 n}=1+\frac{n-k-1}{6 g-3+2 n} .
\end{aligned}
$$

Corollary 9. For any $\left(d_{1}, d_{2}\right) \in \Pi(3 g-2+n, 2)$ and for any $n \in \mathbb{N}$ one has

$$
\varepsilon\left(0^{n-1}, d_{1}, d_{2}\right) \geq-\frac{2}{6 g-1} .
$$

Proof. If one of $d_{1}, d_{2}$ is equal to zero, the statement for arbitrary $n$ follows from (5), so from now on we assume that both $d_{1}, d_{2}$ are strictly positive. For $n=1$ the statement follows directly from (6). This serves us as a base of induction in $n$. Suppose that for all $n=1, \ldots, k$ the statement is true. Let us prove it for $n=k+1$ :

$$
\begin{aligned}
\left\langle\tau_{0}^{k+1} \tau_{d_{1}} \tau_{d_{2}}\right\rangle_{g, k+3} & =\left\langle\tau_{0}^{k} \tau_{d_{1}-1} \tau_{d_{2}}\right\rangle_{g, k+2}+\left\langle\tau_{0}^{k} \tau_{d_{1}} \tau_{d_{2}-1}\right\rangle_{g, k+2} \\
& \geq\left(1-\frac{2}{6 g-1}\right) \cdot\left(\left\lfloor\tau_{0}^{k} \tau_{d_{1}-1} \tau_{d_{2}}\right\rfloor_{g, k+2}+\left\lfloor\tau_{0}^{k} \tau_{d_{1}} \tau_{d_{2}-1}\right\rfloor_{g, k+2}\right) \\
& =\left(1-\frac{2}{6 g-1}\right) \cdot\left(1+\delta_{\text {string }}\left(0^{k+1}, d_{1}, d_{2}\right)\right) \cdot\left\lfloor\tau_{0}^{k+1} \tau_{d_{1}} \tau_{d_{2}}\right\rfloor_{g, k+3} \\
& \geq\left(1-\frac{2}{6 g-1}\right) \cdot\left\lfloor\tau_{0}^{k+1} \tau_{d_{1}} \tau_{d_{2}}\right\rfloor_{g, k+3},
\end{aligned}
$$

where the first equality is the string equation; inequality between the first and the second lines is the assumption of the induction; the equality between the second and the third line is equation (13); the inequality between the third and the forth line is an implication of (14) and of the fact that all the factors in both lines are positive. 
Corollary 10. For any $g, n \in \mathbb{N}$ and for any $\boldsymbol{d} \in \Pi_{0}(3 g-3+n, n)$ one has

$$
1+\varepsilon(\boldsymbol{d}) \geq \lambda(g, 0) .
$$

Proof. Recalling convention (8) for $\lambda(g, 0)$ we conclude that for $n=1$ inequality (16) follows from (3); for $n=2$ inequality (16) follows from (6); for $n \geq 3$ inequality (16) corresponds to $(15)$.

Lemma 11. Let $\boldsymbol{d} \in \Pi(3 g-3+n, n)$. Define $\delta_{\text {dilaton }}(1, \boldsymbol{d})$ by equation

$$
\left\lfloor\tau_{1} \tau_{d_{1}} \cdots \tau_{d_{n}}\right\rfloor_{g, n+1} \cdot\left(1+\delta_{\text {dilaton }}(1, \boldsymbol{d})\right)=(2 g-2+n)\left\lfloor\tau_{d_{1}} \cdots \tau_{d_{n}}\right\rfloor_{g, n} .
$$

Then

$$
\delta_{\text {dilaton }}(1, \boldsymbol{d})=\frac{n-3}{6 g-3+2 n} .
$$

In particular,

$$
\delta_{\text {dilaton }}(1, \boldsymbol{d}) \begin{cases}\geq 0 & \text { when } n \geq 3, \\ =-\frac{1}{6 g+1} & \text { when } n=2, \\ =-\frac{2}{6 g-1} & \text { when } n=1 .\end{cases}
$$

Proof. Dividing both sides of equation (17) by $\left\lfloor\tau_{1} \tau_{d_{1}} \cdots \tau_{d_{n}}\right\rfloor_{g, n+1}$, applying definition (11) and canceling common factors in the numerator and in the denominator of the resulting expression we get

$$
1+\delta_{\text {dilaton }}(1, \boldsymbol{d})=\frac{3(2 g-2+n)}{6 g-3+2 n}=\frac{6 g-6+3 n}{6 g-3+2 n} .
$$

Corollary 12. For any partition $\boldsymbol{d} \in \Pi_{1}(3 g-3+n, n)$ one has

$$
1+\varepsilon(\boldsymbol{d}) \geq\left(1-\frac{1}{6 g+1}\right) \cdot\left(1-\frac{2}{6 g-1}\right) .
$$

Proof. We have seen in (3) that for all 1-correlators we have $\varepsilon(3 g-2)=0$, so for $n=1$ the statement is true. For $n=2$ the statement is a direct implication of equation (6). Suppose that $n \geq 3$.

By Remark 1, the quantity $\varepsilon(\boldsymbol{d})$ does not change under any permutation of the entries of $\boldsymbol{d}$. Thus, we can permute the first $n-2$ elements of the partition without affecting the value of $\varepsilon(\boldsymbol{d})$, in particular, we can place them in the growing order. Since the sum of the first $n-2$ elements is less than or equal to 1 either they are all equal to 0 or they form the sequence $(0, \ldots, 0,1)$ after such reordering. If they all are equal to 0 , the statement follows from equation (15) from Corollary (9).

It remains to consider the case when $n \geq 3$ and when the first $n-2$ elements form a sequence $(0, \ldots, 0,1)$. We prove first the desired inequality for partitions of the form $\left(1, d_{1}, d_{2}\right)$ :

$$
\begin{aligned}
\left\langle\tau_{1} \tau_{d_{1}} \tau_{d_{2}}\right\rangle_{g, 3} & =(2 g) \cdot\left\langle\tau_{d_{1}} \tau_{d_{2}}\right\rangle_{g, 2} \geq\left(1-\frac{2}{6 g-1}\right) \cdot(2 g)\left\lfloor\tau_{d_{1}} \tau_{d_{2}}\right\rfloor_{g, 2} \\
& =\left(1-\frac{2}{6 g-1}\right) \cdot\left\lfloor\tau_{1} \tau_{d_{1}} \tau_{d_{2}}\right\rfloor_{g, 3} \cdot\left(1+\delta_{\text {dilaton }}\left(1, d_{1}, d_{2}\right)\right) \\
& \geq\left(1-\frac{2}{6 g-1}\right)\left(1-\frac{1}{6 g+1}\right) \cdot\left\lfloor\tau_{1} \tau_{d_{1}} \tau_{d_{2}}\right\rfloor_{g, 3} .
\end{aligned}
$$


Here the first equality is the dilaton equation; the inequality which follows is equation (6); the equality between the first two lines is equation (17) and the inequality between the second and the third line is based on equation (18).

To complete the proof of Corollary 12 we prove it for partitions of the form $\left(0^{k+1}, 1, d_{1}, d_{2}\right)$ by induction in $k \geq 0$. The proof follows line-by-line the proof of Corollary 9 .

Lemma 13. Let $\boldsymbol{d} \in \Pi(3 g-3+n-k, n)$, where $k, n \in \mathbb{N}$. Define $\delta_{\text {Virasoro }}(k+1, \boldsymbol{d})$ by equation

$$
\begin{aligned}
& \left\lfloor\tau_{k+1} \tau_{d_{1}} \cdots \tau_{d_{n}}\right\rfloor_{g}\left(1+\delta_{\text {Virasoro }}(k+1, \boldsymbol{d})\right) \\
& =\frac{1}{(2 k+3) ! !}\left(\sum_{j=1}^{n} \frac{\left(2 k+2 d_{j}+1\right) ! !}{\left(2 d_{j}-1\right) ! !}\left\lfloor\tau_{d_{1}} \cdots \tau_{d_{j}+k} \cdots \tau_{d_{n}}\right\rfloor_{g}\right. \\
& \left.\quad+\frac{1}{2} \sum_{\substack{r+s=k-1 \\
r, s \geq 0}}(2 r+1) ! !(2 s+1) ! !\left\lfloor\tau_{r} \tau_{s} \tau_{d_{1}} \cdots \tau_{d_{n}}\right\rfloor_{g-1}\right) .
\end{aligned}
$$

Then

$$
\delta_{\text {Virasoro }}(k+1, \boldsymbol{d})=\frac{n-3}{6 g-3+2 n}-\frac{2 k(2 n-5)}{(6 g-3+2 n)(6 g-5+2 n)} .
$$

Proof. Dividing both sides of equation (20) by $\left\lfloor\tau_{k+1} \tau_{d_{1}} \cdots \tau_{d_{n}}\right\rfloor_{g, n+1}$, applying definition (11) and canceling common factors in the numerator and in the denominator of the resulting expression we get

$$
\begin{aligned}
1+ & \delta_{\text {Virasoro }}(k+1, \boldsymbol{d}) \\
& =\frac{1}{6 g-3+2 n} \cdot\left(\left(\left(2 d_{1}+1\right)+\cdots+\left(2 d_{n}+1\right)\right)+\frac{1}{2} \cdot k \cdot \frac{24 g}{6 g-5+2 n}\right) \\
& =\frac{1}{6 g-3+2 n} \cdot\left((6 g-6+3 n-2 k)+2 k-2 k \cdot \frac{2 n-5}{6 g-5+2 n}\right) \\
& =1+\frac{n-3}{6 g-3+2 n}-\frac{2 k(2 n-5)}{(6 g-3+2 n)(6 g-5+2 n)} .
\end{aligned}
$$

Remark 14. In expression (20) we ignored the third term in the Virasoro constraints. Since this third term is, clearly, positive, this is suitable for getting a lower bound instead of exact asymptotics. It is widely believed that the third term of Virasoro constraints becomes negligible in large genera. We expect that technique from [1] might be useful for replacing the lower bound in (10) by the exact asymptotics under strengthening restrictions on $\alpha$.

We shall need the following technical corollary of Lemma 13.

Corollary 15. Let $k, n$ be integers satisfying $k \geq 0, n \geq 2$. Let $\boldsymbol{d}$ be a partition $\boldsymbol{d} \in \Pi(3 g-3+$ $n-k, n)$, such that $k+1 \leq d_{j}$ for $j=1, \ldots, n-2$, and $k+d_{1}+\cdots+d_{n-2} \leq \frac{3}{2} g$. Then

$$
\delta_{\text {Virasoro }}(k+1, \boldsymbol{d}) \geq-\frac{1}{6 g+1} .
$$

Proof. Use expression (21) for $\delta_{\text {Virasoro }}(k+1, \boldsymbol{d})$. When $n=2$ we get

$$
\delta_{\text {Virasoro }}(k+1, \boldsymbol{d})=-\frac{1}{6 g+1}+\frac{2 k}{(6 g+1)(6 g-1)} \geq-\frac{1}{6 g+1} .
$$

Let $n \geq 3$. By assumption, $(k+1) \leq d_{j}$ for $j=1, \ldots, d_{n-2}$, so $(k+1)(n-2) \leq \frac{3}{2} g$, and hence $2 k(2 n-5)<6 g$, which implies that

$$
\delta_{\text {Virasoro }}((k+1, \boldsymbol{d}))=\frac{n-3}{6 g-3+2 n}-\frac{2 k(2 n-5)}{(6 g-3+2 n)(6 g-5+2 n)}
$$




$$
\geq-\frac{2 k(2 n-5)}{(6 g-3+2 n)(6 g-5+2 n)} \geq-\frac{6 g}{(6 g+3)(6 g+1)}>-\frac{1}{6 g+1} .
$$

Before passing to the step of induction, we recapitulate the properties of the function $\lambda(g, L)$ defined in (7). Recall that the arguments $g, L$ of $\lambda(g, L)$ are nonnegative integers satisfying $g>L$. Inequalities (23)-(25) below follow from the definition of $\lambda(g, L)$. Each inequality is applied to those ordered pairs $g, L$ for which the argument of $\lambda$ on both sides of the inequality belongs to the domain of definition of $\lambda$. We have

$$
1>\lambda(g+1, L)>\lambda(g, L)>\lambda(g, L+1)>0
$$

and

$$
\left(1-\frac{1}{6 g+1}\right) \cdot \lambda(g-1, L-1)=\lambda(g, L)
$$

Combining the latter two relations we get

$$
\begin{aligned}
\left(1-\frac{1}{6 g+1}\right) \cdot \lambda(g, L) & >\left(1-\frac{1}{6(g-1)+1}\right) \cdot \lambda(g, L) \\
& >\left(1-\frac{1}{6(g-1)+1}\right) \cdot \lambda(g-1, L)=\lambda(g, L+1) .
\end{aligned}
$$

Proposition 16 (step of induction). Suppose that for some nonnegative integer $L_{0}$ the following uniform bound is valid: for all integers $g$, $L$ satisfying $g>L_{0}, 0 \leq L \leq L_{0}$, for all partitions $\boldsymbol{d} \in \Pi_{L}(3 g-2+n, n+1)$, where $n \geq 0$, one has

$$
1+\varepsilon(\boldsymbol{d}) \geq \lambda(g, L) .
$$

Then for all integers $g$, $L$ satisfying $g>L_{0}+1,0 \leq L \leq L_{0}+1$, for all partitions $\boldsymbol{d} \in$ $\Pi_{L}(3 g-2+n, n+1)$, where $n \geq 0$, one also has

$$
1+\varepsilon(\boldsymbol{d}) \geq \lambda(g, L)
$$

Proof. We warn the reader that the total number of elements of the partition is denoted in Proposition 16 by $n+1$ and not by $n$ as in Theorem 5 . This allows us to use formulae from the key Lemmas 11 and 13 without adjustments.

By convention $\Pi_{L}(3 g-2,1)=\Pi(3 g-2,1)$ and $\Pi_{L}(3 g-1,2)=\Pi(3 g-1,2)$ for any $L \in \mathbb{Z}_{\geq 0}$. We have seen in (3) that for all 1-correlators we have $\varepsilon(3 g-2)=0$, so for $n=0$ the statement is trivially true. For $n=1$ the statement is a direct implication of inequality (6):

$$
1+\varepsilon(\boldsymbol{d}) \geq\left(1-\frac{2}{6 g-1}\right)=\lambda(g, 0) \geq \lambda(g, L) .
$$

Thus, from now on we can assume that $n \geq 2$. Let $\boldsymbol{d} \in \Pi_{L}(3 g-2+n, n+1)$. If $L \leq L_{0}$, then the statement makes part of the induction assumption. Hence, from now on we can assume that

$$
\boldsymbol{d} \in \Pi_{L_{0}+1}(3 g-2+n, n+1) \backslash \Pi_{L_{0}}(3 g-2+n, n+1),
$$

where $n \geq 2$ and $g>L_{0}+1$. This implies that $d_{1}+\cdots+d_{n-1}=L_{0}+1$ and, hence,

$$
d_{n}+d_{n+1}=3 g-2+n-\left(L_{0}+1\right)>2 L_{0}+2 .
$$

By Remark 1, the quantity $\varepsilon(\boldsymbol{d})$ does not change under any permutation of the entries of $\boldsymbol{d}$. Place to the leftmost position the smallest strictly positive element among the first $n-1$ elements. 
This operation does not change the last two elements of the partition and does not change the sum of its first $n-1$ elements. Denote the resulting partition by $\left(k+1, d_{1}, \ldots, d_{n}\right)$. To prove the proposition we have to prove the inequality

$$
1+\varepsilon\left(k+1, d_{1}, \ldots, d_{n}\right) \geq \lambda\left(g, L_{0}+1\right),
$$

where

$$
\begin{aligned}
& k+d_{1}+d_{2}+\cdots+d_{n-2}=L_{0}, \quad n \geq 2, \quad k \geq 0, \\
& k+1 \leq \min _{\substack{1 \leq i \leq n-2 \\
d_{i}>0}} d_{i}, \quad g>L_{0}+1 \geq 1 .
\end{aligned}
$$

We consider the special case $k=0$ separately. In this special case, when $n=2$ the partition $\left(k+1, d_{1}, \ldots, d_{n}\right)$ becomes $\left(1, d_{1}, d_{2}\right)$ and the desired inequality is proved in (19). Assume that $k=0$ and $n \geq 3$. By (18) we have

$$
\delta_{\text {dilaton }}\left(1, d_{1}, \ldots, d_{n}\right) \geq 0, \quad \text { for } n \geq 3 \text {. }
$$

Thus, for any $g>L_{0}+1$ we have

$$
\begin{aligned}
(1+ & \left.\varepsilon\left(1, d_{1}, \ldots, d_{n}\right)\right) \cdot\left\lfloor\tau_{1} \tau_{d_{1}} \cdots \tau_{d_{n}}\right\rfloor_{g, n+1}=\left\langle\tau_{1} \tau_{d_{1}} \cdots \tau_{d_{n}}\right\rangle_{g, n+1} \\
& =(2 g-2+n)\left\langle\tau_{d_{1}} \cdots \tau_{d_{n}}\right\rangle_{g, n} \geq(2 g-2+n) \cdot\left(\lambda\left(g, L_{0}\right) \cdot\left\lfloor\tau_{d_{1}} \cdots \tau_{d_{n}}\right\rfloor_{g, n}\right) \\
& =\lambda\left(g, L_{0}\right) \cdot\left\lfloor\tau_{1} \tau_{d_{1}} \cdots \tau_{d_{n}}\right\rfloor_{g, n+1} \cdot\left(1+\delta_{\text {dilaton }}(1, \boldsymbol{d})\right) \\
& \geq \lambda\left(g, L_{0}\right) \cdot\left\lfloor\tau_{1} \tau_{d_{1}} \cdots \tau_{d_{n}}\right\rfloor_{g, n+1}>\lambda\left(g, L_{0}+1\right) \cdot\left\lfloor\tau_{1} \tau_{d_{1}} \cdots \tau_{d_{n}}\right\rfloor_{g, n+1} .
\end{aligned}
$$

Here the first equality is definition (12) of $\varepsilon\left(1, d_{1}, \ldots, d_{n}\right)$; the second equality is the string equation (2); the inequality in the middle of the second line is the induction assumption; the equality in the beginning of the third line is definition (17) of $\left(1+\delta_{\text {dilaton }}(1, \boldsymbol{d})\right)$; the inequality in the beginning of the last line is a direct implication of (28); the last inequality is an implication of (23).

Suppose now that $k \geq 1$. We first prove the desired inequality (26) in the special case when

$$
d_{j} \geq 1, \quad \text { for } j=1, \ldots, n-2
$$

and then prove it in the most general situation when some of $d_{j}$ (possibly all of them) are equal to 0 .

Note that by assumption, $k+1$ is less than or equal to any strictly positive element among $d_{1}, \ldots, d_{n-2}$, so inequalities (29), actually, imply that

$$
d_{j} \geq k+1, \quad \text { for } j=1, \ldots, n-2 \text {. }
$$

Also, from (27) we get

$$
k+d_{1}+d_{2}+\cdots+d_{n-2}=L_{0}<\frac{3 g}{2} .
$$

Thus, the partition $\left(k+1, d_{1}, \ldots, d_{n}\right)$ satisfies assumptions of Corollary 15 .

Let $g>L_{0}+1$. Under the above assumptions we have

$$
\begin{gathered}
\left(1+\varepsilon\left(k+1, d_{1}, \ldots, d_{n}\right)\right) \cdot\left\lfloor\tau_{k+1} \tau_{d_{1}} \cdots \tau_{d_{n}}\right\rfloor_{g}=\left\langle\tau_{k+1} \tau_{d_{1}} \cdots \tau_{d_{n}}\right\rangle_{g} \\
\geq \frac{1}{(2 k+3) ! !}\left(\sum_{j=1}^{n} \frac{\left(2 k+2 d_{j}+1\right) ! !}{\left(2 d_{j}-1\right) ! !}\left\langle\tau_{d_{1}} \cdots \tau_{d_{j}+k} \cdots \tau_{d_{n}}\right\rangle_{g}\right.
\end{gathered}
$$




$$
\begin{aligned}
& \left.+\frac{1}{2} \sum_{\substack{r+s=k-1 \\
r, s \geq 0}}(2 r+1) ! !(2 s+1) ! !\left\langle\tau_{r} \tau_{s} \tau_{d_{1}} \cdots \tau_{d_{n}}\right\rangle_{g-1}\right) \\
\geq & \frac{1}{(2 k+3) ! !}\left(\sum_{j=1}^{n-2} \frac{\left(2 k+2 d_{j}+1\right) ! !}{\left(2 d_{j}-1\right) ! !} \cdot \lambda\left(g, L_{0}\right) \cdot\left\lfloor\tau_{d_{1}} \cdots \tau_{d_{j}+k} \cdots \tau_{d_{n}}\right\rfloor_{g}\right. \\
& +\frac{\left(2 k+2 d_{n-1}+1\right) ! !}{\left(2 d_{n-1}-1\right) ! !} \cdot \lambda\left(g, L_{0}-k\right) \cdot\left\lfloor\tau_{d_{1}} \cdots \tau_{d_{n-2}} \tau_{d_{n-1}+k} \tau_{d_{n}}\right\rfloor_{g} \\
& +\frac{\left(2 k+2 d_{n}+1\right) ! !}{\left(2 d_{n}-1\right) ! !} \cdot \lambda\left(g, L_{0}-k\right) \cdot\left\lfloor\tau_{d_{1}} \cdots \tau_{d_{n-1}} \tau_{d_{n}+k}\right\rfloor_{g} \\
& \left.+\frac{1}{2} \sum_{r+s=k-1}(2 r+1) ! !(2 s+1) ! ! \cdot \lambda\left(g-1, L_{0}-1\right) \cdot\left\lfloor\tau_{r} \tau_{s} \tau_{d_{1}} \cdots \tau_{d_{n}}\right\rfloor g-1\right) \\
\geq & \lambda\left(g, L_{0}\right) \cdot\left\lfloor\tau_{k+1} \tau_{d_{1}} \cdots \tau_{d_{n}}\right\rfloor g \\
\geq & \left(1-\frac{1}{6 g+1}\right) \cdot \lambda\left(g, L_{0}\right) \cdot\left\lfloor\tau_{k+1} \tau_{d_{1}} \cdots \tau_{d_{n}}\right\rfloor_{g} \\
\geq & \lambda\left(g, L_{0}+1\right) \cdot\left\lfloor\tau_{k+1} \tau_{d_{1}} \cdots \tau_{d_{n}}\right\rfloor_{g} .
\end{aligned}
$$

Here the first equality is the definition (12) of $\varepsilon\left(1, d_{1}, \ldots, d_{n}\right)$; the first inequality is an instant corollary of the Virasoro constraints in which we omitted the terms in the third line of (1). The second inequality is the induction assumption. The third inequality combines the inequality $\lambda\left(g, L_{0}-k\right)>\lambda\left(g, L_{0}\right)$ which follows from (23), the inequality $\lambda\left(g-1, L_{0}-1\right)>\lambda\left(g, L_{0}\right)$ which follows from $(24)$, and the definition $(20)$ of $\delta_{\text {Virasoro }}(k+1, \boldsymbol{d})$. The inequality $\left(1+\delta_{\text {Virasoro }}(k+\right.$ $1, \boldsymbol{d})) \geq\left(1-\frac{1}{6 g+1}\right)$ is justified by (22). The last inequality is justified in (25).

It remains to prove inequality (26), without extra assumptions (29). In other words, we have to prove the inequality

$$
1+\varepsilon\left(0^{s}, k+1, d_{1}, \ldots, d_{n-s}\right) \geq \lambda\left(g, L_{0}+1\right) .
$$

The case $n-s=0$ follows from (5). For $n-s=1$ inequality (15) implies

$$
1+\varepsilon\left(0^{s}, k+1, d_{1}\right) \geq 1-\frac{2}{6 g-1}=\lambda(g, 0) \geq \lambda\left(g, L_{0}+1\right) .
$$

Thus, we may assume that $n-s \geq 2$ and that the following inequalities are valid:

$$
\left\{\begin{array}{l}
k+d_{1}+d_{2}+\cdots+d_{n-s-2}=L_{0} \\
n \geq s \geq 0 \\
k \geq 1, \\
d_{j}>k \text { for } j=1, \ldots, n-s-2 .
\end{array}\right.
$$

We proceed by induction in $s$. For $s=0$, which serves us as a base of induction, the statement is already proved. We perform a step of induction as follows

$$
\begin{aligned}
(1+\varepsilon & \left.\left(0^{s+1}, k+1, d_{1}, \ldots, d_{n-s}\right)\right) \cdot\left\lfloor\tau_{0}^{s+1} \tau_{k+1} \tau_{d_{1}} \cdots \tau_{d_{n-s}}\right\rfloor_{g}=\left\langle\tau_{0}^{s+1} \tau_{k+1} \tau_{d_{1}} \cdots \tau_{d_{n-s}}\right\rangle_{g} \\
= & \left\langle\tau_{0}^{s} \tau_{k} \tau_{d_{1}} \cdots \tau_{d_{n-s}}\right\rangle_{g}+\left\langle\tau_{0}^{s} \tau_{k+1} \tau_{d_{1}-1} \cdots \tau_{d_{n-s}}\right\rangle_{g}+\cdots+\left\langle\tau_{0}^{s} \tau_{k+1} \tau_{d_{1}} \cdots \tau_{d_{n-s}-1}\right\rangle_{g} \\
= & \left(1+\varepsilon\left(0^{s}, k, d_{1}, \ldots, d_{n-s}\right)\right) \cdot\left\lfloor\tau_{0}^{s} \tau_{k} \tau_{d_{1}} \cdots \tau_{d_{n-s}}\right\rfloor_{g} \\
& +\left(1+\varepsilon\left(0^{s}, k+1, d_{1}-1, \ldots, d_{n-s}\right)\right) \cdot\left\lfloor\tau_{0}^{s} \tau_{k+1} \tau_{d_{1}-1} \cdots \tau_{d_{n-s}}\right\rfloor_{g}+\cdots \\
& +\left(1+\varepsilon\left(0^{s}, k+1, d_{1}, \ldots, d_{n-s}-1\right)\right) \cdot\left\lfloor\tau_{0}^{s} \tau_{k+1} \tau_{d_{1}} \cdots \tau_{d_{n-s}-1}\right\rfloor_{g}
\end{aligned}
$$




$$
\begin{aligned}
\geq & \lambda\left(g, L_{0}\right) \cdot\left\lfloor\tau_{0}^{s} \tau_{k} \tau_{d_{1}} \cdots \tau_{d_{n-s}}\right\rfloor_{g}+\lambda\left(g, L_{0}\right) \cdot\left\lfloor\tau_{0}^{s} \tau_{k+1} \tau_{d_{1}-1} \cdots \tau_{d_{n-s}}\right\rfloor_{g}+\cdots \\
& +\lambda\left(g, L_{0}\right) \cdot\left\lfloor\tau_{0}^{s} \tau_{k+1} \tau_{d_{1}} \cdots \tau_{d_{n-s-2}-1} \tau_{d_{n-s-1}} \tau_{d_{n-s}}\right\rfloor_{g} \\
& +\lambda\left(g, L_{0}+1\right) \cdot\left\lfloor\tau_{0}^{s} \tau_{k+1} \tau_{d_{1}} \cdots \tau_{d_{n-s-2}} \tau_{d_{n-s-1}-1} \tau_{d_{n-s}}\right\rfloor_{g} \\
& +\lambda\left(g, L_{0}+1\right) \cdot\left\lfloor\tau_{0}^{s} \tau_{k+1} \tau_{d_{1}} \cdots \tau_{d_{n-s-2}} \tau_{d_{n-s-1}} \tau_{d_{n-s}-1}\right\rfloor_{g} \\
\geq & \lambda\left(g, L_{0}+1\right)\left(\left\lfloor\tau_{0}^{s} \tau_{k} \tau_{d_{1}} \cdots \tau_{d_{n-s}}\right\rfloor_{g}+\left\lfloor\tau_{0}^{s} \tau_{k+1} \tau_{d_{1}-1} \cdots \tau_{d_{n-s}}\right\rfloor_{g}+\cdots\right. \\
& \left.+\left\lfloor\tau_{0}^{s} \tau_{k+1} \tau_{d_{1}} \cdots \tau_{d_{n-s}-1}\right\rfloor_{g}\right) \\
= & \lambda\left(g, L_{0}+1\right) \cdot\left\lfloor\tau_{0}^{s+1} \tau_{k+1} \tau_{d_{1}} \cdots \tau_{d_{n-s}}\right\rfloor_{g} \cdot\left(1+\delta_{\text {string }}\left(0^{s+1}, k+1, d_{1}, \ldots, d_{n-s}\right)\right) \\
\geq & \lambda\left(g, L_{0}+1\right) \cdot\left\lfloor\tau_{0}^{s+1} \tau_{k+1} \tau_{d_{1}} \cdots \tau_{d_{n-s}}\right\rfloor_{g} .
\end{aligned}
$$

Here the first equality is the definition (12) of $\varepsilon\left(0^{s+1}, k+1, d_{1}, \ldots, d_{n-s}\right)$. The second equality is the string equation (2). (Recall that by convention, if one of $d_{n-s-1}$ or $d_{n-s}$ is equal to zero, the term, containing the negative index $d_{n-s-1}-1$ or $d_{n-s}-1$ respectively, is missing in the string equation and below.) The equality which follows, is equation (12) applied to every term of the resulting expression. The inequality, where $\lambda$ appears on the left-hand side for the first time, is the induction assumption applied to each term. The next inequality follows from the inequality $\lambda\left(g, L_{0}\right)>\lambda\left(g, L_{0}+1\right)$, see (23). The equality which follows is the definition (13) of $\delta_{\text {string }}\left(0^{s+1}, k+1, d_{1}, \ldots, d_{n-s}\right)$. The last inequality is justified by (14).

Proof of Theorem 5. For $L=0$ Theorem 5 follows from Corollary 10. For $L=1$ it follows from (19) combined with the fact that $\left(1-\frac{1}{6 g+1}\right) \cdot\left(1-\frac{2}{6 g-1}\right)>\lambda(g, 1)$. For $L>1$ we apply recursively Proposition 16.

\section{Acknowledgements}

We thank A. Aggarwal for precious comments on the preliminary version of this text, which allowed to correct several misprints and improve the presentation. We are grateful to N. Anantharaman, A. Borodin, D. Chen, M. Kazarian, S. Lando, M. Möller, A. Okounkov, M. Shapiro for stimulating discussions. We thank MPIM in Bonn and MSRI in Berkeley for providing us with excellent working environment. We thank anonymous referees for their generous reports and for helpful suggestions which allowed to improve the presentation. The research of the second author was partially supported by PEPS. The results of Section 1 were obtained at Saint Petersburg State University under support of RSF grant 19-71-30002. This material is based upon work supported by the ANR-19-CE40-0021 grant. It was also supported by the NSF Grant DMS-1440140 while part of the authors were in residence at the MSRI during the Fall 2019 semester.

\section{References}

[1] Aggarwal A., Large genus asymptotics for Siegel-Veech constants, Geom. Funct. Anal. 29 (2019), 1295-1324, arXiv:1810.05227.

[2] Aggarwal A., Large genus asymptotics for volumes of strata of abelian differentials, J. Amer. Math. Soc., to appear, arXiv:1804.05431.

[3] Aggarwal A., Large genus asymptotics for intersection numbers and principal strata volumes of quadratic differentials, arXiv:2004.05042.

[4] Aggarwal A., Delecroix V., Goujard É., Zograf P., Zorich A., Conjectural large genus asymptotics of MasurVeech volumes and of area Siegel-Veech constants of strata of quadratic differentials, Arnold Math. J. 6 (2020), 149-161, arXiv:1912.11702.

[5] Chen D., Möller M., Sauvaget A., Masur-Veech volumes and intersection theory: the principal strata of quadratic differentials (with an appendix by G. Borot, A. Giacchetto, D. Lewanski), arXiv:1912.02267. 
[6] Chen D., Möller M., Sauvaget A., Zagier D., Masur-Veech volumes and intersection theory on moduli spaces of abelian differentials, Invent. Math., to appear, arXiv:1901.01785.

[7] Delecroix V., Goujard É., Zograf P., Zorich A., Masur-Veech volumes, frequencies of simple closed geodesics and intersection numbers of moduli spaces of curves, arXiv:1908.08611.

[8] Delecroix V., Goujard É., Zograf P., Zorich A., Large genus asymptotic geometry of random square-tiled surfaces and of random multicurves, arXiv:2007.04740.

[9] Dijkgraaf R., Intersection theory, integrable hierarchies and topological field theory, in New Symmetry Principles in Quantum Field Theory (Cargèse, 1991), NATO Adv. Sci. Inst. Ser. B Phys., Vol. 295, Plenum, New York, 1992, 95-158, arXiv:hep-th/9201003.

[10] Dijkgraaf R., Verlinde H., Verlinde E., Topological strings in $d<1$, Nuclear Phys. B 352 (1991), 59-86.

[11] Eskin A., Okounkov A., Pillowcases and quasimodular forms, in Algebraic Geometry and Number Theory, Progr. Math., Vol. 253, Birkhäuser Boston, Boston, MA, 2006, 1-25, arXiv:math.DS/0505545.

[12] Eskin A., Zorich A., Volumes of strata of Abelian differentials and Siegel-Veech constants in large genera, Arnold Math. J. 1 (2015), 481-488, arXiv:1507.05296.

[13] Goujard É., Volumes of strata of moduli spaces of quadratic differentials: getting explicit values, Ann. Inst. Fourier (Grenoble) 66 (2016), 2203-2251, arXiv:1501.01611.

[14] Kazarian M., Recursion for Masur-Veech volumes of moduli spaces of quadratic differentials, J. Inst. Math. Jussieu, to appear, arXiv:1912.10422.

[15] Kazarian M., Lando S., An algebro-geometric proof of Witten's conjecture, J. Amer. Math. Soc. 20 (2007), 1079-1089, arXiv:math.AG/0601760.

[16] Kontsevich M., Intersection theory on the moduli space of curves and the matrix Airy function, Comm. Math. Phys. 147 (1992), 1-23.

[17] Liu K., Xu H., A remark on Mirzakhani's asymptotic formulae, Asian J. Math. 18 (2014), 29-52, arXiv:1103.5136.

[18] Mirzakhani M., Simple geodesics and Weil-Petersson volumes of moduli spaces of bordered Riemann surfaces, Invent. Math. 167 (2007), 179-222.

[19] Mirzakhani M., Weil-Petersson volumes and intersection theory on the moduli space of curves, J. Amer. Math. Soc. 20 (2007), 1-23.

[20] Okounkov A., Pandharipande R., Gromov-Witten theory, Hurwitz numbers, and matrix models, in Algebraic Geometry - Seattle 2005, Part 1, Proc. Sympos. Pure Math., Vol. 80, Amer. Math. Soc., Providence, RI, 2009, 325-414, arXiv:math.AG/0101147.

[21] Witten E., Two-dimensional gravity and intersection theory on moduli space, in Surveys in Differential Geometry (Cambridge, MA, 1990), Lehigh University, Bethlehem, PA, 1991, 243-310.

[22] Yang D., Zagier D., Zhang Y., Masur-Veech volumes of quadratic differentials and their asymptotics, arXiv:2005.02275.

[23] Zograf P.G., An explicit formula for Witten's 2-correlators, J. Math. Sci. 240 (2019), 535-538. 